\title{
Adaptive Robust Video Watermarking Scheme based on Dual Transform Domains and Holography
}

\author{
DaYou Jiang ${ }^{1}, \mathrm{De}_{\mathrm{Li}^{1}}{ }^{*}$ and Jong Weon $\mathrm{Kim}^{2}$ \\ ${ }^{1}$ Department of Computer Science, Yanbian University \\ 133002, Yanji, China \\ [e-mail: ybdxgxy13529@163.com,leader1223@ybu.edu.cn] \\ ${ }^{2}$ Department of Intellectual Property, Sangmyung University \\ 110743, Seoul, Korea \\ [e-mail:jwkim@smu.ac.kr] \\ *Corresponding author: De Li
}

\begin{abstract}
This paper proposes a digital video watermarking scheme based on 2D-DWT and pseudo 3D-DCT transformation, otherwise Singular Value Decomposition (SVD) are applied. To enhance the robustness of watermark, Particle Swarm Optimization (PSO) algorithm and Holography are used for embedding watermarks. The Hash scrambling transformation is used for ensuring the security of the method. During the embedding process, the quantization coefficients are dynamically determined by PSO algorithm. The method selects some keyframes in raw video to extract the luminance components and take them into some groups. The subband of generated hologram watermark is embedded into the subband of the singular value of each DCT block in every group. The experimental are conducted to verify the robustness through some common signal processing and frame-based attacks.
\end{abstract}

Keywords: video watermarking, Hash scrambling transformation, Holography,, 2D-DWT and pseudo 3D-DCT, Singular Value Decomposition

\section{Introduction}

Digital watermarking technique is an effective method to solve the copyright protection problems of digital media. Most of the current video watermarking technique insert watermark directly to uncompressed or compressed video sequences [1]. Niu and Sun proposed a wavelet based watermarking method that embeds decomposed watermark at different resolution in the corresponding resolution of the decomposed video by means of multirate solution signal decomposition [2]. Barni, et al., proposed a robust watermarking scheme for raw video that alters the DFT coefficients of the brightness components of the tobe-marked frames. The scheme is robust against filtering, scaling and cropping attacks [3]. Th. Rupachandra, et al., proposed a video watermarking scheme based on visual cryptography, scene change detection and DWT. The scheme uses an identical sub-band watermark for the same scene, but different parts in different scenes [4]. Semin Kim, et al., proposed a novel modality fusion method designed for combining spatial and temporal fingerprint information to improve video copy detection performance [5]. Antonio Cedillo-Hrenanderz, et al., proposed a transcoding resilient watermarking scheme based on spatio-temporal HVS and DCT. The quantization index modulation (QIM) algorithm is used to embed and detect the watermark in 2D-DCT domain [6]. Marwen Hasnaoui, et al., proposed a multiple-symbol 
QIM video watermarking; the paper introduces the theoretical framework allowing for the binary QIM embedding techniques to be extended towards multiple-symbol QIM [7].

In this paper, we proposed a new digital video watermarking scheme based on dual transform domains and holography. The holography has high shear resistance characteristic so that the method is robust to cropping attack. In order to improve the transparency and the imperceptibility of the watermarked video, the watermark is hidden into the low frequency of the luminance components of the raw video and the quantization coefficients are dynamically determined by using PSO. The Hash scrambling transformation is used for achieving the high security of the watermark.

The rest of the paper is organized as follows: in section 2, hash scrambling transformation, 2D-DWT and pseudo 3D-DCT transformation and holography are briefly proved. Details of the proposed embedding and extracting scheme are described in Section 3. Section 4 presents a variety of simulation experimental results, which illustrate the effectiveness of the proposed algorithm. Finally we conclude the paper in Section 5.

\section{Background}

In this section, the concepts of Hash scrambling transformation, 2D-DWT and pseudo 3DDCT and hologram are briefly described.

\subsection{Hash Scrambling Transformation}

Hash scrambling transformation has properties of none-collision, great none-crack and strong key-control. In this paper, we apply hash scrambling transformation to original watermark to ensure the security of the method. Various hash algorithms are available such as MD5 (Message Digest 5) and SHA-2 (Security Hash Algorithm) which hash data strings, thus changing their state from being natural to seemingly unnatural state. A hash function is more formally defined as the mapping of bit strings of an arbitrary finite length to strings of fixed length [8]. In the paper, we use MD5 hash algorithm to produce a new pseudo random generators.

The transformations need five parameters. The watermark matrix, the quantity of the total pixels and three secrete keys: key1, key2 and key3. The output parameters are column number and row number [9].

\subsection{D-DWT and pseudo 3D-DCT}

Considering that the DWT preserves the local edges and noise reduction in the low frequency domain after the image decomposition. While the DCT is obviously to decrease the correlation of the video image. In this paper, we combined the DWT and DCT to process video image. The principle of 2D DWT and pseudo 3D DCT is shown in Figure 1. Firstly, we choose key-frame of the raw video sequence and separate them into groups, and each group consists of $\mathrm{N}$ frames. Secondly, we select the $\mathrm{Y}$ sample of each frame and take 2D-DWT to each frame of every group. Then divide the LL coefficients into $8 \times 8$ blocks of all frames. At last, take pseudo 3D-DCT transform to each blocks of the group. 


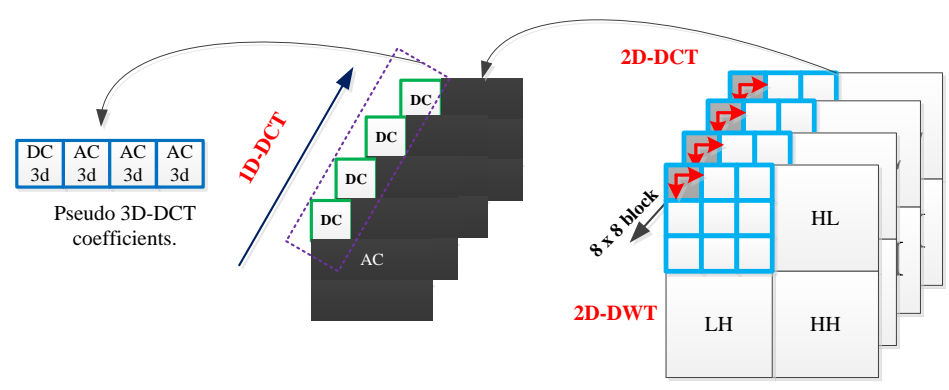

Figure 1. The Principle of 2D-DWT and Pseudo 3D-DCT

\subsection{Holography}

The holography concept was first proposed by Dennis Gabor in 1947. The lens-less Fourier transform hologram [10] is employed in the paper. Supposing that the object point source and the reference point source are on the same plane namely $x 0 y 0$ plane. The CCD plane is named $x y$ plane. The recording distance between the two planes is $d$. Suppose the complex amplitude of the object is $O_{0}(x 0, y 0)$ and the reference point source is $(-b, 0)$.

According to the Fresnel diffraction theory and ignoring the constant factor, the complex amplitude of the object wave in the CCD plane is defined

$$
O(x, y)=\iint_{\infty} O_{0}(x, y) \exp \left\{\frac{j k}{2 d}\left[\left(x-x_{0}\right)^{2}+\left(y-y_{0}\right)^{2}\right]\right\} d x_{0} d y_{0},
$$

The complex amplitude of the reference wave in the CCD plane is defined

$$
R(x, y)=\exp \left[\frac{j k}{2 d}\left(x^{2}+y^{2}\right)\right] \exp \left[-\frac{j k}{d}\left(x x_{r}+y y_{r}\right)\right],
$$

The intensity distribution of the interference pattern in the hologram plane is

$$
H(x, y)=|O+R|=|O|^{2}+|R|^{2}+O^{*} R+O R^{*},
$$

Where $*$ denotes the complex conjugate.

Hologram is usually as watermark data is embedded into the cover image, for improving the performance of the conventional watermarking system against some attacks such as noise, cropping, and so on. In the paper, we apply the holography to the scrambling watermark to generate the hologram image and then add it into singular values of the raw video.

\subsection{Particle Swarm Optimization Algorithm}

The PSO algorithm which based on the observation of a simplified social model of bird flocks is an evolutionary computation technology, proposed by Dr. Eberhart and Dr. Kennedy in 1995 [11]. In the PSO algorithm, each solution of optimization problem can be called a particle. All particles find the optimal solution through iteration. In every iteration, particles update their own position by tracking two extremums. One is the individual extremum pBest, another one is the global extremum $g$ Best.

When the pBest and gBest are found, each particle will adjust its flight speed and the new location according to equation (4) and (5).

$$
\begin{gathered}
v^{\prime}=w^{*} v+c 1 * r 1 *(\text { pBest }- \text { present })+c 2 * r 2 *(g B \text { est }- \text { present }) \\
\text { present } t^{\prime}=\text { present }+v^{\prime}
\end{gathered}
$$

Where $v$ and $v^{\prime}$ denote the initial and updated respectively, present and present'stand for the initial and updated new position respectively. The weight $\mathrm{w}$ controls the particles velocity of each generation, and the acceleration weights $c 1$ and $c 2$ represent the two random values 
for not jumping into local optimum. The weights $r 1$ and $r 2$ denote two uniformly distributed random values. In the algorithm, inertia weight $w$, maximum moving speed $V_{\max }$, and acceleration weights $c 1, c 2$, these parameters are set based on empirical value.

In the paper, we employ $P S O$ algorithm to find the best quantization coefficients that use for watermark embedding process.

\section{Proposed Zero Video Watermarking Scheme}

\subsection{Watermark Embedding Process}

Figure 2 shows the process of the embedding scheme. The main steps of the embedding procedure are described as follow:

Step1: Watermark image preconditioning

(1) Select the binary image with $18 \times 22$ pixels as watermark information.

(2) Apply Hash scrambling transformation described in 2.1 to the watermark with key one.

(3) Apply Hologram to scrambling watermark in order to generate hologram watermark, then divided it into 16 sub-band watermarks.

Step2: YUV video preconditioning

(1) Select 256 frames of the raw YUV video,then select key-frames in every 4 frames and separate each 4 key-frames into groups.

(2) Select Y samples of each key-frame of each group. Then apply 2D-DWT and pseudo 3D-DCT described in 2.2 and SVD in orderly to those frames in each group to get the first singular values $\mathrm{V}$, then save theses values as key 2 .

Step3: Multiply sub-band hologram watermarks by quantization coefficients generated by using PSO method, then add this watermarks' coefficients to the correspondent singular values to replace the original singular values.

Step4: Generate DCT coefficients by applying reverse SVD transform, then applying reverse 2D-DCT and reverse 2D-DWT in orderly to restore $\mathrm{Y}$ samples of each frame of each group.

Step5: Restore watermarked video sequences.

\subsection{Watermark Extraction Process}

The extraction process is similarity to the embedding process. Figure 3 shows the process of the extraction scheme. The main steps of the extracting procedure are described as follow:

Step1: Select 256 frames of the watermarked YUV video, then select key-frames in every 4 frames and separate each 4 key-frames into groups.

Step2: Select Y samples of each key-frame of each group. Then apply 2D-DWT and pseudo 3D-DCT described in 2.2 and SVD in orderly to those frames in each group to get the first singular values $\mathrm{V}^{\prime}$.

Step3: Subtract key 2 values from correspondent singular values $V^{\prime}$, then divide the singular values after subtracting by quantization coefficients which saved as key 3 . The values are sub-band hologram watermarks.

Step4: Apply reverse hologram to those hologram watermarks. Then apply reverse Hash scrambling transformation on scrambling watermark to get the extracted watermark. 


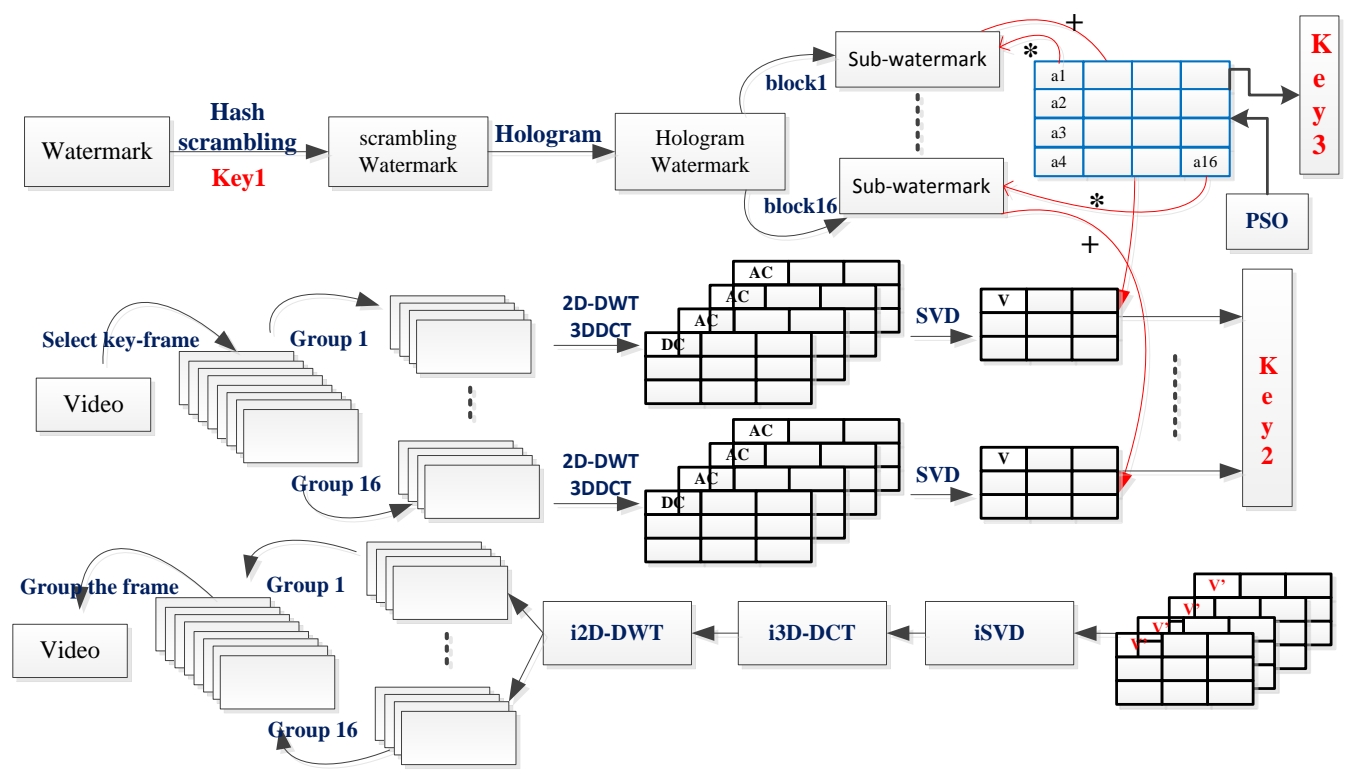

Figure 2. Process of the Embedding Scheme

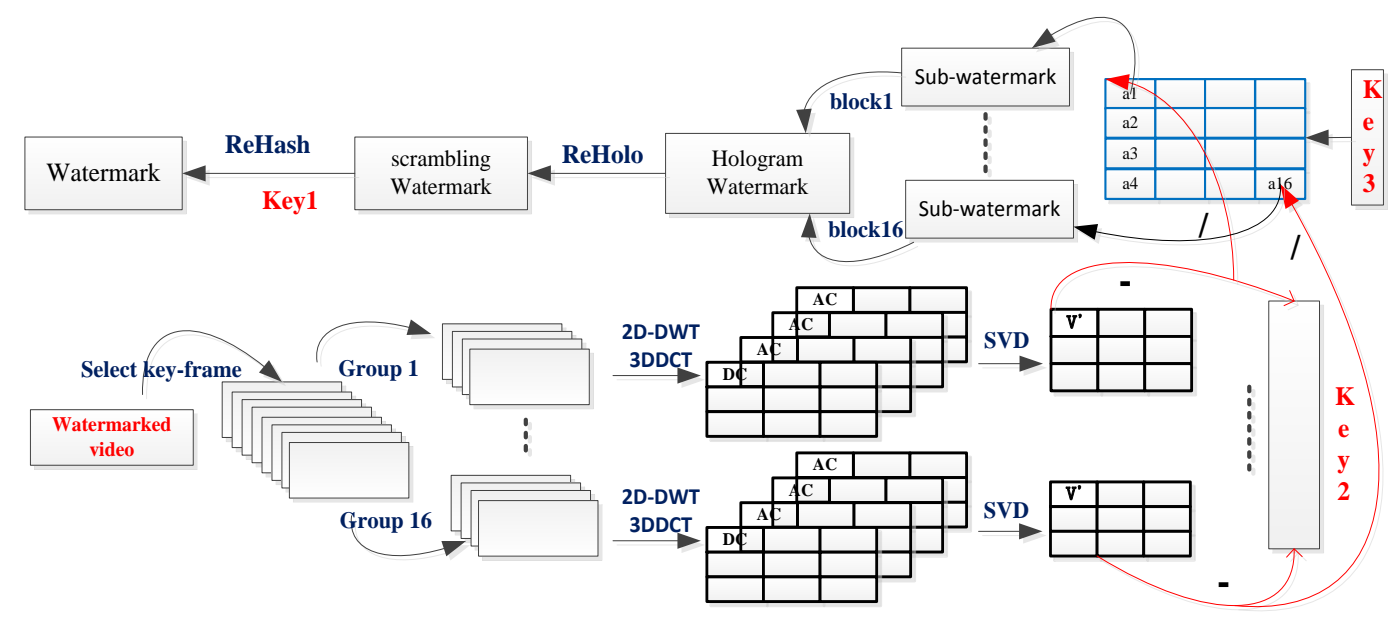

Figure 3. Process of the Extraction Scheme

\section{Experiment Results and Analysis}

To evaluate the performance of the proposed scheme, we used 4 video sequences with CIF format and 30 FPS. All video sequences have 300 frames which are available in [12]. The watermark image is denoted by a binary image, the size of which is $18 \times 22$.

In this paper, the peak signal-to-noise ratio (PSNR) and mean squared error (MSE) are used to measure the imperceptibility of watermarking. Bit Error Rate (BER) and normalized cross-correlation (NC) are used to evaluate the similarity between an original watermarks Wand the extracted watermark. 
Since the video has lots of frames, so we take the average PSNR to measure the imperceptible of the watermarking method. Table 1 shows watermark and video sequences used for evaluation of the proposed method.

Table 1. The Video Sequences and Watermark Image

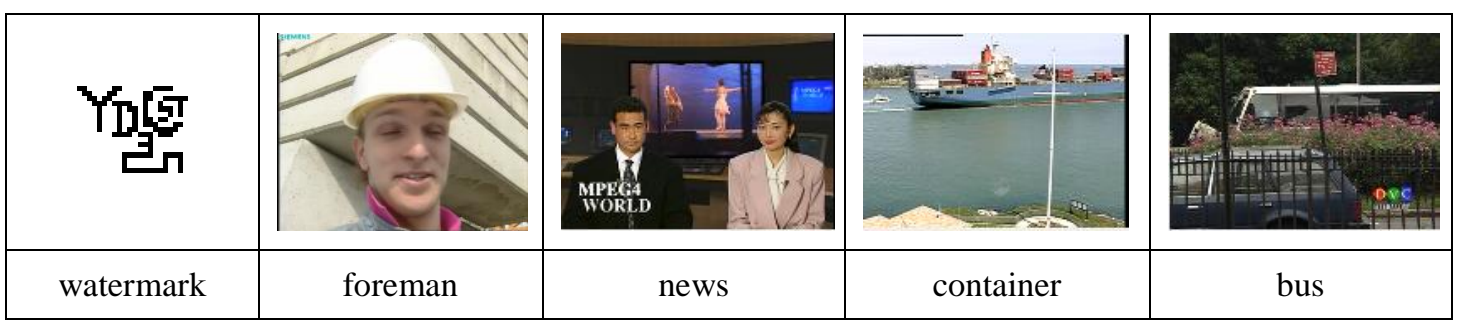

The PSNR values of different types of video files are more than $44 \mathrm{~dB}$, and the difference between the watermarked video and the cover video is unperceived. In addition, the NC is 1 ; the BER is 0 . It is evident that the proposed algorithm can accurately extract the watermark without error.

In order to assess the embedded watermark robustness, the watermark video sequences are attacked using some common signal processing and frame-based attacks. In the proposed method, the watermark is embedded into Y samples of each key-frame with static step size of the video scene. All the attacks are performed in these key-frames.

The watermarked video sequences are subject to signal process attacks including noise contamination such as Gaussian noise attack (shown in Table 2) and salt \& pepper noise (shown in Table 2), Gaussian low-pass filtering ,median filtering, averaging filtering (shown in Table 4),rotation(shown in Table 5) and cropping (shown in Table 6).

Additionally we evaluated the proposed scheme against frame-based attacks. A video sequence contains lots of temporal redundancy, so frame-based attacks, such as frame dropping (shown in Table 7), frame averaging (shown in Table 8) and frame swapping(shown in Table 9) are efficiently done to remove the watermark sequence without cause significant quality degradation.

Table 2 and Table 3 show the result of proposed method under noise contamination. We set Gaussian noise at zero mean and its variances ranging from 0.005 to 0.02 . The salt \& pepper noise density is set ranging from 0.01 to 0.04 . It is found that the watermark is clearly identifiable. It is because the high frequency components are affected by the noise, while the low frequency components in LL sub band is the least affected.

Table 4 shows the result of proposed method under different filtering such as Gaussian low-pass filtering, median filtering and averaging filtering. The values of window sizes are all set $3 \times 3$. The result shows that the proposed method is highly robust to filtering attacks.

Table 5 shows the result of the proposed method under rotation attack at different rotation angle from $10^{\circ}$ to $60^{\circ}$. In order to make sure the watermark is identifiable, we rotate the video in counter clockwise direction firstly and then rotate the returned video in clockwise direction at the same angle. So the BER value is totally determined by cropping region, because we applied hologram in the method, the method is robust under cropping attack. That is to say, the method is robust under rotation attack.

Table 6 shows the result of the proposed method under cropping attack. The percentage of cropping is ranging from $12.5 \%$ to $50 \%$. $12.5 \%$ cropping corresponds to removing 36 horizontal lines per frame in the video. The results show that the method is robust under cropping attack. 
Table 7 shows the result of the proposed method under frame dropping attack. In the method, the dropping key-frames are selected from each group. The number of groups is ranging from 3 to 15 . The results show that the BER value is less than 0.03 .

Table 8 shows the result of the proposed method under frame averaging attack. In the method, the averaging key-frames are selected from each key-frame. The number of frames is ranging from 6 to 16 (25\% percentage of all key-frames). We collect a number of frames, then replacing each pixel' value of each frame by the estimated average value of each pixel. The results show that the method is robust under frame averaging attack.

Table 9 show the result of the proposed method under frame swapping attack. Since the watermark is embedded into the group of frames, the frame swapping within-group has no effect on the BER value, so that we doing the inter-group swapping program. In the experiment, the key-frames are selected from each group firstly, then select contiguous groups to swap. In the program, the number 5 of swapping frames means that 10 frames from different 10 groups are swapped. Even though, the watermark is clearly identifiable.

Table 2. Extracted Results of Gaussian Noise Attacks

\begin{tabular}{|c|c|c|c|c|}
\hline variance & 0.005 & 0.010 & 0.015 & 0.020 \\
\hline $\begin{array}{l}\text { Attacked } \\
\text { Frame }\end{array}$ & & & & \\
\hline $\begin{array}{l}\text { Extracted } \\
\text { Watermark }\end{array}$ & $\mathrm{YDg}_{\mathrm{B}}$ & Yूg & $\mathrm{Y}_{\mathrm{DS}}$ & YSg \\
\hline $\mathrm{NC}$ & 0.9965 & 0.9947 & 0.9965 & 0.9876 \\
\hline BER & 0.0051 & 0.0076 & 0.0051 & 0.0177 \\
\hline
\end{tabular}

Table 3. Extracted Results of Salt \& Pepper Noise Sttacks

\begin{tabular}{|c|c|c|c|c|}
\hline density & 0.01 & 0.02 & 0.03 & 0.04 \\
\hline $\begin{array}{l}\text { Attacked } \\
\text { Frame }\end{array}$ & & & & \\
\hline $\begin{array}{c}\text { Extracted } \\
\text { Watermark }\end{array}$ & Y⿰氵巨⿹ & Yפg & Ygg & Y. \\
\hline $\mathrm{NC}$ & 1 & 0.9965 & 0.9947 & 0.9840 \\
\hline BER & 0 & 0.0051 & 0.0076 & 0.0227 \\
\hline
\end{tabular}


Table 4. Extracted Results of Filtering

\begin{tabular}{|c|c|c|c|}
\hline type & Gaussian low-pass filtering & Averaging filtering & Median filtering \\
\hline $\begin{array}{l}\text { Extracted } \\
\text { Watermark }\end{array}$ & Yots & Yפ & YDIS \\
\hline $\mathrm{NC}$ & 0.9965 & 0.9911 & 1 \\
\hline BER & 0.0051 & 0.0126 & 0 \\
\hline
\end{tabular}

Table 5. Extracted Results of Rotation Attacks

\begin{tabular}{|c|c|c|c|c|}
\hline angle & 10 & 30 & 45 & 60 \\
\hline $\begin{array}{l}\text { Attacked } \\
\text { Frame }\end{array}$ & & & & \\
\hline $\begin{array}{c}\text { Extracted } \\
\text { Watermark }\end{array}$ & YDS & . & YDS & Yogs \\
\hline $\mathrm{NC}$ & 0.9982 & 0.9893 & 0.9929 & 0.9965 \\
\hline BER & 0.0225 & 0.0152 & 0.0101 & 0.0051 \\
\hline
\end{tabular}

Table 6. Extracted Results of Crop Attacks

\begin{tabular}{|c|c|c|c|c|}
\hline percentage & Crop $12.5 \%$ & Crop 25\% & Crop $37.5 \%$ & Crop 50\% \\
\hline $\begin{array}{l}\text { Attacked } \\
\text { Frame }\end{array}$ & & & & \\
\hline $\begin{array}{l}\text { Extracted } \\
\text { Watermark }\end{array}$ & YDE & Y. & 伽焉 & רן \\
\hline $\mathrm{NC}$ & 0.9911 & 0.9840 & 0.9714 & 0.9641 \\
\hline BER & 0.0126 & 0.0227 & 0.0404 & 0.0505 \\
\hline
\end{tabular}

Table 7. Extracted Results of Frame Dropping

\begin{tabular}{|c|c|c|c|c|}
\hline group & $1-3$ & $1 \sim 7$ & $1 \sim 11$ & $1 \sim 15$ \\
\hline $\begin{array}{l}\text { Extracted } \\
\text { Watermark }\end{array}$ & Y鸨 & YDS & Ysig & 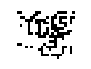 \\
\hline $\mathrm{NC}$ & 0.9982 & 0.9830 & 0.9830 & 0.9775 \\
\hline BER & 0.0025 & 0.0234 & 0.0234 & 0.0309 \\
\hline
\end{tabular}


Table 8. Extracted Results of Frame Averaging

\begin{tabular}{|c|c|c|c|c|}
\hline numbers & 6 & 9 & 12 & 16 \\
\hline $\begin{array}{c}\text { Extracted } \\
\text { Watermark }\end{array}$ & YDS & YDS & YDS & YDST \\
\hline $\mathrm{NC}$ & 0.9982 & 0.9911 & 0.9822 & 0.9696 \\
\hline BER & 0.0225 & 0.0126 & 0.0253 & 0.0707 \\
\hline
\end{tabular}

Table 9. Extracted Results of Frame Swapping (Inter-group Swapping)

\begin{tabular}{|c|c|c|c|c|}
\hline inter-group & 2 & 3 & 4 & 5 \\
\hline $\begin{array}{c}\text { Extracted } \\
\text { Watermark }\end{array}$ & YDE & 照 & 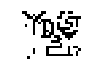 & 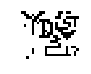 \\
\hline $\mathrm{NC}$ & 0.9822 & 0.9625 & 0.9642 & 0.8963 \\
\hline BER & 0.0253 & 0.0530 & 0.0505 & 0.1435 \\
\hline
\end{tabular}

\section{Conclusion}

In this paper, we proposed a video watermarking technique robust against several signal processing distortions and frame-based attacks. To improve robustness and security of the method, the hash scrambling transformation and hologram are used. During watermark embedding, we apply hash scrambling transformation to scrambling the watermark and generate it to hologram watermarks firstly. Then we apply 2D-DWT and pseudo 3D-DCT, and SVD in orderly to the raw video. Finally we add the hologram watermarks into singular values to hide the information. During the watermark extraction, we do some of the corresponding reverse transformation to get the watermark. Although the proposed scheme still has limitations such as a need for keys. But it is blind and also has high imperceptibility and robustness.

\section{ACKNOWLEDGEMENTS}

This research project was supported by the National Natural Science Foundation of China (Grant No. 61262090)

\section{Reference}

[1] G. Doerr and J. Dugelay, "A guide tour if video watermarking", Signal Processing Image Communication, vol. 18, no. 4, (2003), pp. 263-282.

[2] X. Niu and S. Sun, "A new wavelet based digital watermarking for video", In Proc. IEEE Digital Signal Processing Workshop, (2003) October.

[3] M. Barni, F. Bartolini, R. Caldelli, A. D. Rosa and A. Piva, "A robust watermarking approach for raw video", In Proc. $10^{\text {th }}$ International Packet Video Workshop, PV2000, (2000).

[4] T. R. Singh, K. M. Singh and S. Roy, "Video watermarking scheme based on visual cryptography and scene change detection", International Journal of Electronics and Communications (AEU), vol. 67, (2013), pp. 645-651.

[5] K. Semin, J. Y. Choi, H. Seungwan and M. R. Yong, "Adaptive weighted fusion with new spatial and temporal fingerprint for improved video copy detection”, Signal Processing Image Communication, (2014), http://dx.doi.org/10.1016/j.image.2014.05.002. 
[6] C. H. Antonio, C. H. Manuel, G. V. Mireya, N. M. Mariko, P. M. Hector and R. A. Alejandro, "Transcoding resilient video watermarking scheme based on spatio-temporal HVS and DCT", Signal Processing, vol. 97, (2014), pp. 40-50.

[7] H. Marwen and M. Mihai, "Multiple-symbol QIM video watermarking", Signal Processing Image Communication, vol. 29, (2014), pp. 107-127.

[8] C. Abbas, C. Joan, C. Kevin and M. Paul, "A hash-based image encryption algorithm", Optics Communications, vol. 283, (2010), pp. 879-893.

[9] G. P. Du and X. Y. Cai, "The algorithm of watermarking by hash disturbing", Journal of Yunnan University, vol. 31, no. S1, (2009), pp. 234-236.

[10] H. Cui, D. Y. Wang, Y. X. Wang, J. Zhao and Y. Z. Zhang, "Phase Contrast Imaging of Biologic Cells based on Lensless Fourier Transform Digital Holography", First International Conference on Cellular, Molecular Biology, Biophysics and Bioengineering(CMBB), (2010), pp. 303-306.

[11] M. Alfredo and S. Valentino, "Optimizing Web Content Presentation: A Online PSO Approach", 2009IEEE/WIC/ACM International Conference on Web Intelligence and Intelligent Agent Technology. Milan, Italy, vol. 3, (2009), pp. 26-29.

[12] <http://trace.eas.asu.edu/yuv/index.html>.

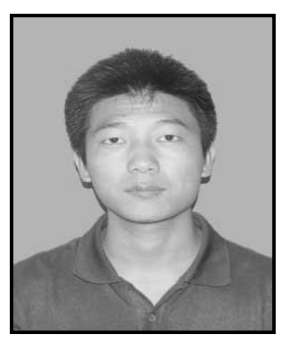

DaYou Jiang, is a postgraduate, major in Information Security, now studying at Yanbian University in China. His research interests are in the areas of copyright protection technology, information security, digital watermarking.

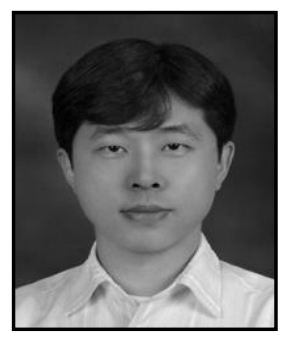

De $\mathbf{L i}$, received the Ph.D. degree from Sangmyung University, major in computer science in 2005. He is currently a professor of Dept. of Computer Science at Yanbian University in China. He is also a Principal Researcher at Copyright Protection Research Institute, Sangmyung University. His research interests are in the areas of copyright protection technology, digital watermarking, and digital forensic marking.

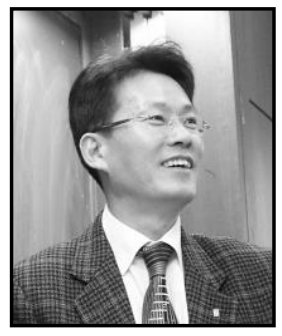

JongWeon Kim, received the Ph.D. degree from University of Seoul, major in signal processing in 1995 . He is currently a professor of Dept. of Intellectual Property at Sangmyung University in Korea. He has a lot of practical experiences in the digital signal processing and copyright protection technology in the institutional, the industrial, and academic environments. His research interests are in the areas of copyright protection technology, digital rights management, digital watermarking, and digital forensic marking. 